La revue La revue pour l'histoire du CNRS

POUR L'HISTOIRE DU CNRS

13 | 2005

Regards sur des laboratoires en sciences humaines et sociales

\title{
Un regard sur le Centre d'ethnologie française
}

Martine Segalen 1

\section{OpenEdition}

Journals

Édition électronique

URL : https://journals.openedition.org/histoire-cnrs/1683

DOI : 10.4000/histoire-cnrs. 1683

ISSN : 1955-2408

Éditeur

CNRS Éditions

Édition imprimée

Date de publication : 3 novembre 2005

ISBN : 978-2-271-06349-6

ISSN : $1298-9800$

Référence électronique

Martine Segalen1, «Un regard sur le Centre d'ethnologie française », La revue pour I'histoire du CNRS

[En ligne], 13 | 2005, mis en ligne le 03 novembre 2007, consulté le 20 mai 2021. URL : http://

journals.openedition.org/histoire-cnrs/1683; DOI : https://doi.org/10.4000/histoire-cnrs. 1683

Ce document a été généré automatiquement le 20 mai 2021.

Comité pour l'histoire du CNRS 


\title{
Un regard sur le Centre d'ethnologie française
}

\author{
Martine Segalen1
}

Le Centre d'ethnologie française, un des premiers laboratoires associés du CNRS en sciences humaines, est dissous fin 2005. Martine Segalen y fut recrutée comme chercheur en 1972 et elle en fut le directeur de 1986 à 1996. Ce laboratoire était associé au Musée national des arts et traditions populaires qui ferme également ses portes en septembre 2005. L'auteur pose ici son regard sur cette institution, fondée sur une étude des archives et sa propre expérience, par définition subjective ${ }^{2}$.

2 Au Musée de l'Homme, aux côtés du Dr Paul Rivet, puis au Musée national des arts populaires dont il est nommé conservateur en 1937, Georges-Henri Rivière s'assigne pour but de faire de l'ethnologie une science, appuyée sur une muséologie totalement renouvelée.

3 En 1938, à l'ouverture du musée, un grand colloque de folklore - qui se voulait la «science du peuple» - rassembla des chercheurs qui n'étaient pas encore des ethnologues puisque la discipline en tant que telle n'existait pas, mais des historiens, des géographes, des folkloristes. Le premier projet de musée n'ayant pu se réaliser avant la guerre, on sait que Georges-Henri Rivière, à la faveur de la proximité entre les valeurs de Vichy et le champ du folklore, dirigea une série d'enquêtes sur l'architecture rurale, le mobilier, le calendrier traditionnel qui contribuèrent à asseoir la vocation scientifique du musée. Celle-ci s'affirmera lentement après la guerre, alors que Georges-Henri Rivière se bat sur tous les fronts pour obtenir la construction d'un nouvel édifice qui abritera les collections qu'il constitue patiemment. L'argumentaire de la mise en valeur d'un patrimoine témoin d'un ordre passé sera relayé dans les années 1960 par un argumentaire scientifique.

4 Dès les débuts du musée, la présence de membres dépendant du jeune Centre national de la recherche scientifique ne pouvait que conforter l'image que le fondateur construisait de son institution en devenir. Georges-Henri Rivière développa la métaphore de musée-laboratoire, qui caractérisa l'institution et contribua à sa réputation, bien avant l'ouverture des portes du musée au public en 1972. En 1966 est 
signé le premier contrat entre le CNRS et le musée (représenté par la Direction des musées de France) pour marquer la fusion des deux institutions.

Mais des éléments de disjonction déjà sensibles, avant même la mise en place du Centre d'ethnologie française, se cristallisèrent dans les années 1980, participant à la crise de l'institution muséale. Ce musée-laboratoire tant vanté a abrité une illusion, un malentendu dont ces quelques pages tentent de décrire les linéaments.

Liste du personnel au 29 octobre 1937

Personnel scientifique :

Conservateur: Georges-Henri Rivière, ancien sous-directeur du musée d'ethnographie du Trocadéro

Conservateur adjoint: André Varagnac, professeur d'enseignement secondaire en congé, diplômé d'études supérieures de philosophie

Attachée : Agnès Humbert, diplômée de l'École du Louvre

Sept chargés de mission des musées nationaux (sans traitement) collaborent dans le département :

Louise Alcan, licenciée ès lettres, diplômée d'études supérieures d'histoire et de géographie, diplômée de l'Institut d'art et d'archéologie ;

Denise Sussfeld, licenciée ès lettres, diplômée d'études supérieures de philosophie ;

Pierre-Louis Duchartre, folkloriste ;

René Laporte, écrivain ;

Marc Leproux, instituteur ;

Guy Pison, architecte DPLG, ancien pensionnaire de la Casa Vélasquez à Madrid.

En outre, l'assemblée permanente des présidents des chambres d'agriculture a institué, par décision en date du 22 octobre 1937, un chargé de mission auprès du département des Arts et traditions populaires, à charge qu'il poursuive des études de métayage considérées sous leur aspect folklorique. Ce poste a été confié à Marcel Maget, diplômé d'études supérieures de philosophie. À cette liste des premiers collaborateurs, il faut ajouter les noms de Louis Dumont qui viendra rejoindre l'équipe du musée en 1938 et Suzanne Tardieu en 1940.

Un homme, un musée ou l'émergence de l'ethnologie scientifique

6 Au lendemain de la guerre, le Musée national des arts et traditions populaires et la science ethnologique ne faisaient qu'un. La valeur des enquêtes conduites dans le cadre des chantiers intellectuels pendant l'Occupation auréolait la jeune institution, mais dans cette période de vaches maigres, il fallait se contenter d'enquêtes plus ponctuelles. Les membres de l'équipe conduisaient, selon le vocabulaire en cours, des "prospections", brefs séjours de terrain auprès d'informateurs institutionnels ou sélectionnés par le biais de réseau de correspondants locaux installés en province. Le résultat de ces enquêtes, documentations, collectes, était rassemblé dans les sous-sols qu'occupait le musée dans l'aile Paris du Palais de Chaillot. Ces travaux étaient conduits dans le cadre du Laboratoire d'ethnographie française, dirigé par Marcel Maget, de 1948 à 1962, qui, malgré l'absence de structure officielle, posait les bases d'enquêtes solides et systématiques. Au terme de "folklore», plus ou moins compromis avec Vichy, était substitué celui «d'ethnographie métropolitaine », puis «française », plus scientifique.

7 Le champ était alors clair : il s'agissait d'étudier la civilisation traditionnelle rurale, d'en recueillir les derniers feux, à l'aide de missions sur le terrain et en suivant le modèle des monographies d'objet très détaillées que Marcel Maget ${ }^{3}$ avait mis au point. La culture matérielle était la principale cible des recherches, ainsi que l'art populaire et les manifestations sociales (fêtes, calendrier traditionnel). Recherche, collecte, documentation des objets et présentation muséographique s'articulaient 
harmonieusement, comme en témoignent les nombreuses expositions temporaires que Georges-Henri Rivière organisa tant que le nouveau bâtiment n'était pas en voie d'achèvement proche, par exemple les expositions relatives aux objets domestiques, arts du cirque, jeux de force et d'adresse, coiffes de France, bergers de France, etc.

Vers une ethnologie du contemporain

8 Au début des années 1960, le CNRS lançait les Recherches coopératives sur programme (RCP) qui devaient associer, autour de problématiques communes, des chercheurs issus de plusieurs disciplines. Après l'enquête pluridisciplinaire qui fut conduite à Plozévet, sous la houlette du Dr Robert Gessain, directeur du Musée de l'Homme, Georges-Henri Rivière entreprit de diriger, avec André Leroi-Gourhan, une grande enquête en Aubrac, qui se déroula sur quatre années (1964-1968). Il s'agissait d'étudier « un établissement humain " caractérisé par une économie d'élevage à vocation fromagère qui s'était constituée dans la seconde moitié du XIX ${ }^{\mathrm{e}}$ siècle. Décrire le tableau complet des activités techniques, sociales et culturelles des communes occupant les hauts plateaux d'altitude situés à l'intersection du Cantal, du Lot et de l'Aveyron, tel était l'objectif. Les nombreux chercheurs, ethnologues, linguistes, historiens, agronomes, sociologues, etc. devaient aussi rendre compte des changements socio-économiques liés à l'Europe agricole du premier marché commun qui conduisait les éleveurs à abandonner leurs fromageries d'altitude (les burons) et à transformer, par le miracle de l'insémination par des taureaux charolais, leurs maigres vaches Aubrac en bovins pour la production de viande.

9 Certes, l'intérêt de Georges-Henri Rivière fut toujours vif pour toutes les manifestations sociales qui caractérisaient son temps, cependant il s'agissait le plus souvent de collectes de circonstance ${ }^{4}$. Avec l'Aubrac, l'occasion lui fut donnée de dire définitivement adieu au folklore. Une ethnologie très neuve fut ainsi développée, s'intéressant à l'histoire de la mise en place de l'économie fromagère et de son déclin, aux mouvements migratoires, aux influences réciproques entre villages et villes vers lesquelles migraient les Aubraciens.

Le CNRS assura le financement des missions puis tint la promesse faite à Georges-Henri Rivière de publier la totalité des enquêtes dans une publication coordonnée comportant huit volumes ${ }^{5}$, qui démarra en 1970 et s'acheva en 1986, un an après sa mort.

11 Moment historique de l'émergence d'une ethnologie scientifique et d'une alliance étroite et unique entre recherche, collecte et muséographie, cette enquête allait donner ses lettres de noblesse à l'ethnologie française au sein de l'anthropologie sociale. Dans un rapport destiné à un membre de la commission du $\mathrm{CNRS}^{6}$, Georges-Henri Rivière remarquait: "Cette entreprise marque une étape très importante du développement de notre discipline. Elle souligne dans notre domaine, comme le font parallèlement d'autres branches de l'ethnologie, que notre science n'a pas pour seule mission de sauver des patrimoines en voie de disparition, mais que, engagée dans les problèmes du présent, elle contribue à une prospective de l'homme». La grande "entreprise Aubrac " comme on l'appelait avait compté pour beaucoup dans le processus qui conduisit le CNRS à s'allier étroitement au musée. Elle fut également une expérience unique puisque les chercheurs assumèrent toutes les tâches, depuis l'enquête de terrain, la collecte, la documentation jusqu'à l'élaboration des vitrines, sous la direction de Georges-Henri Rivière. Une telle cohésion ne se retrouvera plus par la suite.

Un bâtiment moderne pour une ethnologie scientifique 
12 Le projet de nouveau bâtiment, conçu par Jean Dubuisson offrait un écrin architectural à l'alliance entre réserves d'objets, salles d'expositions, salles de documentation, bibliothèque, services de restauration, photographique, muséographique et bureaux pour le personnel des chercheurs et des conservateurs. Lorsque Les Lettres françaises, le 2 mars 1961, font l'éloge du projet: «Un musée révolutionnaire est en train d'élever lentement ses huit étages de fer, de béton et de verre. Pour la première fois en France se construit un complexe muséographique imaginé et calculé point par point selon les perspectives les plus avancées de la science », on peut comprendre que la « science " à laquelle l'article fait référence est celle de l'architecture, mais aussi de l'ethnologie moderne.

13 Avec ses deux galeries, galerie d'étude au sous-sol destinée à un public de spécialistes, galerie culturelle au rez-de-chaussée destinée à tous les publics, surmontées d'une tour de verre abritant les services, l'édifice est pensé par Jean Dubuisson et Georges-Henri Rivière comme une sorte de temple dont l'ascension conduit aux espaces les plus nobles, ceux de la recherche, ou comme le dit Georges-Henri Rivière répondant à un journaliste du Monde en février 1972: "Le profane doué de curiosité devient un chercheur en puissance, une vocation en devenir. [...] Celui-ci passera ainsi s'il en est digne, du narthex à la nef et à la cella. Ainsi le musée sera-t-il en même temps un instrument de promotion culturelle et scientifique.»

Un laboratoire au musée : rencontre et désaccord de deux corps professionnelsLe soutien croissant du CNRS

Dès ses débuts avant la guerre, le CNRS fut partenaire du musée, fournissant au coup par coup soit des moyens en missions, soit des allocations pour des projets de recherche. Ainsi la célèbre mission consacrée à la musique folklorique de BasseBretagne de 1938 fut financée par la Caisse nationale de la recherche scientifique; pendant la guerre, plusieurs membres du musée bénéficièrent de ses allocations. De plus, il abondait le budget d'équipement, autorisant l'achat d'appareils photographiques ou destinés à réaliser des enregistrements sonores. Le CNRS accordait des postes de chercheurs, et c'était là une manne institutionnelle à laquelle le musée recourut, car il n'existait alors aucun corps de conservateurs spécialisés en ethnologie et la Direction des musées de France n'attribuait que très chichement des postes.

15 En 1943, Claudie Marcel-Dubois, la première ethnomusicologue comme on la nomma après la guerre, fut nommée chargée de recherches (alors deuxième grade dans la carrière), de même que Suzanne Tardieu, en 1946. Maguy Pichonnet-Andral, qui travailla aux côtés de Claudie Marcel-Dubois commença comme stagiaire de recherches la même année, puis d'autres chercheurs vinrent les rejoindre dans la seconde moitié des années 1950. Aucun d'entre eux n'avait véritablement de formation ethnologique; ils venaient d'horizons divers, la géographie pour Mariel Jean-Brunhes Delamarre, la littérature pour Marie-Louise Tenèze, etc. Faute de personnel relevant du ministère de la Culture suffisant, le schéma institutionnel élaboré par Georges-Henri Rivière consistait à confier à ces chercheurs tout à la fois l'aspect recherche et l'aspect scientifico-administratif de la collecte, la gestion des collections et la mise en exposition. En somme, il s'agissait d'établir une continuité entre le travail scientifique de recherche, de collecte et son aboutissement au musée, dans les circuits du catalogage, de la restauration et de l'exposition. Symbiose qui fut tenue jusqu'à l'ouverture du musée, comme en témoignent les expositions temporaires que GeorgesHenri Rivière et son équipe renouvelaient régulièrement pour ne pas se faire oublier 
des institutions de tutelle et comme en témoigne aussi l'enquête Aubrac qui, outre sa publication, a considérablement enrichi les fonds du musée.

À partir de 1955, le nombre des personnels CNRS $^{7}$ se développa. Tout en conduisant leurs travaux personnels, ceux-ci s'engageaient, par une sorte de contrat moral, à consacrer une partie de leur temps à des "travaux d'intérêt collectif » exercés en principe le plus possible selon leur spécialisation ${ }^{8}$. Depuis le milieu des années 1950, alors que Georges-Henri Rivière se plaignait d'un manque chronique de personnel, quelques postes de conservateurs avaient cependant été attribués au musée. Ceux-ci étaient responsables de secteurs fondamentaux, mais non directement liés à la recherche (mise en place des galeries, inventaire des collections, bibliothèque, secrétariat administratif) ; la plupart d'ailleurs acquirent une compétence d'ethnologue en suivant les cours de Georges-Henri Rivière. En 1959, on comptait trois conservateurs, neuf chercheurs ou techniciens CNRS. Dans les années 1960, c'est vers le CNRS, alors en plein développement et relativement généreux, que Georges-Henri Rivière se tournait pour essayer de résoudre les besoins criants en personnel de l'institution'.

Dès lors, la recherche prit le pas sur toutes les autres considérations, comme le proclame Georges-Henri Rivière lors d'un congrès international : "La recherche est l'infrastructure de toute notre institution. Elle constitue notre contribution à l'avancement de l'ethnologie, elle conditionne le rassemblement des objets et des documents, elle inspire l'action éducative et culturelle. Nous sommes, en définitive, un musée-laboratoire et cela explique que le Centre national de la recherche scientifique nous donne son appui $»^{10}$. De plus, la recherche n'était plus seulement orientée vers la connaissance des techniques; elle s'intéressait aux "structures sociales, aux représentations ${ }^{11} »$. La science au service de l'ethnologie l'était aussi au service de la muséologie. On sait que Georges-Henri Rivière a inventé une muséographie neuve, tranchant avec les anciennes présentations, qui associait harmonieusement la rigueur scientifique à l'esthétique.

L'infléchissement vers la recherche eut une double conséquence vis-à-vis de la Direction des musées de France au sein du ministère de la Culture : au plan interne, ses personnels se trouvèrent souvent rejetés vers des tâches administratives et techniques ; au plan institutionnel, la tutelle, organisée autour des Beaux-Arts, ne comprenait pas la nature de la démarche ethnologique, le temps qu'elle nécessite, les aléas qui lui sont liés.

Déjà des tensions au sein de la maison

19 En 1963, l'organigramme du musée comporte ainsi deux structures, les services d'une part (photothèque, phonothèque, bibliothèque, laboratoire, collections, etc..) et les départements d'autre part, qui couvraient les douze secteurs scientifiques: artisanat, costume, coutumes et croyances, danse, équipement domestique, esthétique, ethnomusicologie, histoire, jeux, littérature, techniques d'acquisition et de production, structures socio-économiques. Comme l'indique leur nom, les services sont au service... de la science. De plus, Georges-Henri Rivière nourrissait une illusion : couvrir tous les champs du social, les découper en domaines à la tête desquels serait placé, si possible, un tandem constitué d'un chercheur et d'un conservateur, ou sinon l'un ou l'autre.

Liste du personnel en 1957

Personnels musées de France: Marcel Maget, Pierre-Louis Duchartre, Michèle Richet et Hélène Tremaud (Marcel Maget démissionnera de ses fonctions le 1er février 1962). 
Personnels CNRS: Suzanne Tardieu, attachée, chef du service des collections, Claudie Marcel-Dubois, maître de recherches, (thèse d'Université annoncée sur les instruments de musique populaire), Marguerite Pichonnet-Andral, attachée, Louise Alcan, attachée, Marie-Louise Tenèze attachée, Isaac Chiva, attaché de recherches, assistant de Marcel Maget, Clémence Duprat, collaborateur technique, comme Pierre Soulier.

Selon l'ancien système qui s'était incarné dans les chantiers intellectuels, on trouve des personnels rémunérés au titre des chômeurs intellectuels, Mlles Gracy, Demoinet, Duclaux, Château.

Enfin, d'autres agents sont rétribués à titre privé : Melle Clavé (bibliothèque) ; Mariel Jean Brunhes Delamarre, vacataire depuis le 25 juin, spécialiste de l'outillage agricole et pastoral traditionnels (" en espérant un recrutement par le CNRS »).

Deux chargés de mission : Jacques Barré et André Lagrange.

André Desvallées est nommé assistant conservateur en 1959. C'est lui qui va suivre la programmation du nouveau siège.

Toute tendue vers l'ouverture du nouveau siège au bois de Boulogne, l'équipe travaillait en bonne entente sous la férule exigeante de son charismatique et dictatorial patron, mais des tensions étaient déjà palpables et Georges-Henri Rivière les évoquait dans son rapport de 1964 : «L'inégalité des contributions risque d'affaiblir l'amalgame que nous avons voulu réaliser, elle rend plus difficile à nos conservateurs et à nos assistants la démonstration de leurs capacités scientifiques, elle peut développer en eux un sentiment de frustration ${ }^{12}$.»

Au début des années 1970, il renouvelait son souhait que les chercheurs fussent associés autant que possible aux activités culturelles de l'établissement. Il anticipait la rancœur que les conservateurs pourraient ressentir si les chercheurs s'attribuaient exclusivement la part de la recherche, reléguant leurs collègues du musée au simple rang de gestionnaires de collections.

La création du Centre d'ethnologie française du Musée des arts et traditions populaires

Le CNRS fonctionnait avec des commissions disciplinaires décidant des recrutements et carrières des personnels, ainsi que de l'allocation des moyens. Tout en menant son épuisant combat pour l'achèvement de son cher musée, Georges-Henri Rivière, promu au grade de conservateur en chef le 13 avril 1962 et toujours fin stratège, se fit élire à la commission compétente, alors section 20 (anthropologie, préhistoire, ethnologie), en 1957, puis en $1963^{13}$ afin d'être au cœur du dispositif. Et avec succès, puisqu'en 1964, il pouvait écrire que « 7 sur 12 des départements sont dirigés par des chercheurs CNRSArts et traditions populaires (CNRS-ATP), 10 des 18 membres des départements sont des agents CNRS». L'équipe CNRS des ATP qui préparait les collectes, conduisait des enquêtes, participait à la programmation scientifique du nouveau siège ${ }^{14}$ trouvait aussi son compte dans le musée, dans le cadre, écrit Georges-Henri Rivière, « de leur contrat de bonne volonté »: «Ils sont les utilisateurs privilégiés des ressources du musée, les départements qu'ils gèrent constituent pour eux des observatoires aux larges perspectives, ils se sentent les membres d'une communauté de travail ».

Placé au centre du système, Georges-Henri Rivière sût se saisir de l'opportunité ouverte par la création de structures mixtes de laboratoires associés à diverses institutions scientifiques ou d'enseignement.

24 À la demande d'un ethnologue africaniste, Jean-Paul Lebeuf, qui travaillait à l'université de Paris X Nanterre dans le jeune département d'ethnologie fondé par Éric de Dampierre, Georges-Henri Rivière fournit en janvier 1965 un rapport détaillé des liens entre le Musée et la recherche autour de trois axes: le laboratoire, les 
publications, la RCP Aubrac. Et pour mieux convaincre ses interlocuteurs que les ATP étaient débarrassés de tout folklore passéiste, il insistait sur l'inflexion du domaine : «L'ethnologie de la France, désormais, s'axe sur le passage de la société traditionnelle à la société industrielle, constitue sur la première de vastes archives écrites audiovisuelles et coopère avec d'autres disciplines - notamment l'histoire et la sociologie, l'anthropologie sociale et l'agronomie - en vue d'une connaissance rétrospective et prospective de notre pays ${ }^{15}$ ».

Fin connaisseur des rouages administratifs du CNRS et en étroite complicité avec son directeur général, Georges-Henri Rivière inscrivit donc le musée dans la première vague de créations de musées-laboratoire ${ }^{16}$. Restait la question de son nom: la commission exprima «le souhait que sa dénomination soit modifiée et corresponde d'une façon plus précise aux recherches effectuées par [les] équipes ».

Le document original stipule donc : «Art. 1er [Ici sont couverts de croix à la machine la formule: "Le laboratoire du Musée des ATP » et à la place figure] Le Centre d'ethnologie française est associé au CNRS pour la période du 1er janvier 1966 au 31 décembre 1969 ». Ce document fut signé par Pierre Monbeig ${ }^{17}$, directeur adjoint du CNRS pour les lettres et sciences humaines, par Jean Châtelain, directeur des musées de France et par Georges-Henri Rivière.

Entre musée et recherche : un écart qui se creuse

La création du musée-laboratoire donna à l'institution muséale un très grand prestige, d'autant plus que c'était jusque dans les années 1970 le seul lieu d'une élaboration scientifique de l'ethnologie française; de plus, elle facilita la vie administrative par la mise à disposition de crédits réguliers et d'aides multiples. Alors que le musée allait enfin s'ouvrir, la recherche connaissait son plein essor. Le laboratoire partagea les quelques années de gloire dont le «Louvre du peuple» devait jouir. La presse servait sans cesse la référence à l'influence de Claude Lévi-Strauss dont on disait qu'il avait dessiné le plan de la galerie culturelle. Les médias comme le public furent au rendezvous jusqu'à la fin des années 1970 .

Nouvelles thématiques de recherche, un éloignement avec le musée

28 Mais de quelle recherche s'agissait-il ? Dans les années 1950 et 1960, les ethnologueschercheurs du musée des ATP inventèrent une nouvelle ethnologie, abandonnant les grandes enquêtes extensives qui avaient caractérisé les années d'entre les deux guerres et de la guerre au profit de travaux de terrain intensifs. Mais une fois les collectes terminées, pour la plupart d'entre eux, le travail consistait souvent à établir des classifications, inspirées par les travaux d'André Leroi-Gourhan, qui servirent de base aux présentations muséographiques, notamment dans la galerie d'étude. Ce parti pris classificatoire convenait bien aux expositions d'objets relatifs aux chaînes opératoires de l'agriculture, de l'élevage, de la viticulture ou de la vie domestique. Mais il trouvait ses limites dès lors qu'on s'intéressait aux faits sociaux, présentés selon d'autres propositions : les âges de la vie pour le costume, le cycle musical des fêtes profanes et religieuses, etc.

Dès le milieu des années 1970, sous l'influence de l'ethnologie exotique, une nouvelle génération de chercheurs développa des travaux qui changèrent d'échelle, mais aussi de paradigmes et d'objet ${ }^{18}$. Soudain, les sujets de prédilection des ATP, liés essentiellement aux systèmes techniques, apparaissaient «vieillots ». En témoigne le manque d'intérêt avec lequel fut accueillie la publication Aubrac, lorsque lentement, un à un, ses huit volumes furent publiés. Le religieux, la parenté, la sorcellerie, l'identité, 
l'urbain supplantaient les anciens thèmes de recherche : c'était une sorte de revanche du social sur le technique.

Avec l'arrivée du nouveau conservateur du musée-laboratoire, Jean Cuisenier, en 1968, les jeunes chercheurs que la commission de sociologie affecta à l'institution inscrivirent leurs travaux dans ces nouveaux courants, participant au développement d'une ethnologie du "réel », attentive aux changements de la société contemporaine et à ses enjeux. Les commissions compétentes, au fil des années, jugèrent ces travaux satisfaisants, en prenant bien soin de ne jamais porter de jugement sur les activités plus proprement muséales.

31 Cependant, le paysage institutionnel changeait sensiblement : le laboratoire n'était plus l'unique lieu de fabrication de l'ethnologie scientifique. Liés à des universités ou à l'École pratique des hautes études (qui devait en partie devenir École des hautes études en sciences sociales, EHESS), plusieurs centres de recherche parisiens ou en région témoignaient de la vitalité de l'ethnologie française ; ils contribuèrent indirectement à la dissociation entre musée et recherche comme au rapprochement entre enseignement et recherche, fabriquant du même coup des ethnologues peu familiers des problématiques muséales. Alors que l'ethnologie française se renouvelait profondément, celle des ATP, dans son lien intime avec les objets et les présentations muséales, se trouvait contestée.

De plus, l'institution fut caractérisée par une discontinuité thématique flagrante liée à la procédure de recrutement du CNRS, fondée sur la valeur d'un projet scientifique personnel et non sur l'inscription dans la continuité de tel ou tel secteur de recherche préexistant. Ainsi la belle illusion des départements censés couvrir la totalité du champ social se révéla crûment : il n'y avait personne pour recueillir le savoir de la vieille équipe. Peut-on imaginer le département d'égyptologie du musée du Louvre tomber en déshérence? Tel fut le cas pour la littérature orale, la danse ou encore les objets domestiques une fois que les fondateurs eurent pris leur retraite. Les structures du CNRS n'avaient pas les moyens (ou le goût) de s'interroger sur la pertinence des thématiques de la maison dont le devenir institutionnel lui échappait. L'arrivée d'autres chercheurs contribua à diversifier les orientations et les objets de recherche et à creuser l'écart entre le musée et le CNRS. Le problème resta latent pour exploser publiquement lors de la crise de 1990. Ainsi la discontinuité de la transmission du savoir était patente. De plus, les nouveaux thèmes de l'ethnologie, tournée maintenant vers l'étude des identités, de la parenté, du religieux, du symbolique, etc. se prêtaient mal à collectes et expositions. Alors que les thèmes de recherche de l'équipe fondatrice portaient sur une société rurale, vue essentiellement à travers le prisme de ses activités matérielles et sociales, ceux de la nouvelle équipe portaient sur la société contemporaine qu'on ne pouvait qu'imparfaitement documenter à travers ses objets.

Toutefois, loin de se désintéresser du musée, la plupart des chercheurs et ITA du CNRS furent sensibles à l'étonnante richesse, diversité et qualité des collections; outre la publication de leurs recherches, ils produisirent un grand nombre des expositions temporaires qui furent présentées régulièrement à partir de 1972; ces expositions furent d'ailleurs le seul moyen d'attirer régulièrement du public au musée puisque les galeries permanentes ne furent jamais renouvelées, contrairement au vœu du fondateur qui avait voulu des espaces flexibles.

34 Paradoxalement, dans les années 1970 mais aussi par la suite, les chercheurs CNRS ne cherchaient pas à valoriser ce travail avec le musée auprès de leur institution de tutelle 
et ne mentionnaient pas toujours dans leur rapport d'activité les tâches accomplies car - disait-on - ce serait pénalisé par la commission!

La production scientifique fut considérable, Jean Cuisenier ayant lancé plusieurs collections d'ouvrages exploitant les fonds du musée. Par ailleurs, il rajeunit la vieille revue Arts et traditions populaires, qui, sous les habits neufs d'Ethnologie française, a contribué fondamentalement aux progrès de la recherche dans le domaine.

Et pourtant, dès le milieu des années 1980, le désenchantement était perceptible. Le musée ne trouvait plus son public et la Direction des musées de France lui opposait le succès des jeunes écomusées. Celle-ci avait d'ailleurs créé au sein de la direction du patrimoine une petite structure dynamique, la Mission du patrimoine ethnologique, qui se donnait pour tâche de penser l'ethnologie de la France et disposait de nombreux moyens. Celle-ci relayait en quelque sorte le projet initial porté par le Centre d'ethnologie française qui rencontrait ses limites en termes de moyens, d'entreprises collectives. Jamais le CNRS, au sein de sa commission compétente, ne développa de réflexion sur l'ethnologie de la France, désormais pilotée à partir d'un ministère qui, paradoxe supplémentaire, laissait en déshérence le Musée qu'elle avait ouvert pour en traiter!

Dans ce contexte général, le déséquilibre entre recherche et conservation, les querelles institutionnelles entre musée et laboratoire concernant les statuts des différents acteurs du musée et leur rôle au sein de l'établissement prirent une acuité nouvelle avec l'arrivée d'une jeune génération de conservateurs.

Des conservateurs du CNRS?

Il n'existait pas de conservateurs spécialistes du domaine jusqu'à ce que la Direction des musées de France mette en place une procédure de recrutement destinée à de jeunes diplômés de l'Université en ethnologie ou en histoire de l'art, ayant suivi une série de stages pratiques leur permettant d'être inscrit sur la liste dite «d'aptitude aux fonctions de conservateur ». Alors que les anciens prenaient leur retraite, quelques jeunes arrivèrent, mettant en œuvre leurs compétences dans un secteur spécifique. Pas plus que pour les chercheurs, il n'y eut véritablement transmission des savoirs. Louise Alcan, responsable du secteur costumes, avait concentré son action sur les costumes de région: arrivée dans le département, une jeune conservatrice commença, elle, une collecte de costumes de travail, depuis les tenues classiques des bouchers jusqu'aux combinaisons en papier tissé dont on vêt le personnel qui nettoie le métro: collecte intéressante mais qui s'arrêta dès lors que celle-ci quitta l'établissement ${ }^{19}$. L'examen de la constitution des collections permet aujourd'hui de dissiper l'illusion de collections totales, car elles sont souvent le fruit de l'intérêt passager de certains pour tel ou tel sujet.

Liste des personnels au 30 juin 1981

Chercheurs et collaborateurs de recherche CNRS-DMF (Direction des musées de France) et assimilés :

Guy Barbichon (CNRS), Marion Boudignon-Hamon (CNRS), Yvonne Broutin (CNRS), Jacques Cheyronnaud (DMF), Elizabeth Claverie (CNRS), Gérard Collomb (CNRS), Jean Cuisenier (CNRS), Geneviève Delbos (CNRS), Jacqueline Demoinet (DMF), JeanFrançois Depelsenaire (CNRS), Marie-Thérèse Duflos (CNRS), Sylvie Forestier (DMF), Aliette Geistdoerfer (CNRS), Denise Gluck (CNRS), Jean-François Gossiaux (EHESS), Marie-France Gueusquin (CNRS), Alain Guey (CNRS), Jean Guibal (DMF), Martine Jaoul (DMF), Pierre Labat (CNRS), Donatien Laurent (CNRS), Françoise Lautman (CNRS), Thérèse Léon (CNRS), Françoise Loux (CNRS), Claudie Marcel-Dubois (CNRS), Elizabeth Margerie (DMF), Chantal Martinet (DMF), Claude Michelat (CNRS), 
Alain Monestier (DMF), Jacques Nicourt (DMF), Jacques Pasquet (CNRS), André Pelle (CNRS), Gisèle Perez-Borie (CNRS), Aline Petit (CNRS), Marie-Marguerite PichonnetAndral (CNRS), Philippe Pigelet (CNRS), Marie-Christine Pouchelle (CNRS), Patrick Prado (CNRS), Henri Raulin (CNRS), Philippe Richard (MSH), Aline Ripert (CNRS), Claude Royer (CNRS), Michèle Salitot (CNRS), Arlette Schweitz (CNRS), Martine Segalen (CNRS), Colette Sluys (CNRS), Suzanne Tardieu-Dumont (CNRS), MarieLouise Tenèze (CNRS), Henriette Touillier-Feyrabend (CNRS), Tran QuangHai (CNRS), Suzanne Vachette (CNRS), Geneviève Vayssière (CNRS), Thierry Voinier (CNRS)

6 doctorants auxquels s'ajoutent 33 personnes de statut divers répertoriés comme « personnel DMF, EHESS et assimilé concourant aux opérations de recherche ».

Et encore 8 personnels CNRS de l'antenne de Marseille.

André Desvallées a quitté le musée en 1977.

Ces jeunes conservateurs revendiquaient à juste titre leur place au sein d'une institution dont ils avaient, en tout premier lieu, la responsabilité: les tâches techniques liées à la conservation des objets absorbaient une grande partie de leur temps. Leur statut comportait aussi explicitement une mission de recherche, mais le contenu de cette recherche s'écartait de plus en plus de ce qu'était le programme des chercheurs : elle était tournée vers des thématiques appuyées sur des collectes d'objets. Les conservateurs étaient donc doublement en porte-à-faux, vis-à-vis d'une direction muséale qui n'attachait guère d'intérêt à leur travail et vis-à-vis du CNRS, incarnant « la recherche ", mais pas celle qu'ils conduisaient, puisque dans ces années-là, la culture matérielle n'intéressait guère de monde dans les instances des commissions.

Par ailleurs, le déséquilibre numérique entre les deux corps ne cessa de s'accroître, la pénurie de conservateurs s'aggravant à partir de janvier 1991, lorsque, au système de la liste d'aptitude qui témoignait des capacités acquises par les postulants lors de leurs stages en musée, fut substitué un concours national pour accéder au nouveau corps des " conservateurs du patrimoine». Si quelques nouveaux conservateurs rejoignirent les équipes du musée, ce fut pour conforter le clivage avec la recherche CNRS, arc-boutés qu'ils étaient sur les collections, ce que d'ailleurs la Direction des musées de France leur direction - leur recommandait.

Ces divergences et ces insatisfactions provoquaient des tiraillements au quotidien, signes révélateurs d'un conflit qui fut plus ou moins ouvert selon les périodes (et le point de vue du conservateur en chef), mais demeura latent, tant que Jean Cuisenier assura la double fonction de conservateur et directeur du laboratoire, pour se révéler dans toute son ampleur lorsque les deux fonctions furent dissociées en 1986.

Pour conclure

4 En dépit des conflits internes, liés en partie aux différents statuts, mais surtout à l'errance dans lequel le projet muséal ATP était tombé, il régnait une ambiance très chaleureuse dans la maison à laquelle la plupart était très attachés; les amitiés et les connivences dépassaient d'ailleurs le clivage chercheur-conservateur et dépendaient plutôt de l'ancienneté dans la maison, du projet intellectuel de chacun. La relation des personnels à l'institution était affective et explique le grand malaise qui s'est emparé d'eux lorsque la « chute de la maison Rivière » devint évidente.

Il appartiendra de mesurer les responsabilités de l'État, à travers ses organes de recherche et culturels, dans la chronique de cette mort annoncée. L'État culturel s'est désintéressé d'un patrimoine qui n'incarnait pas l'identité de la France, même s'il était présenté dans un musée "national»; les instances de la recherche, rendues 
impuissantes par leurs structures mêmes, observaient les chercheurs quitter les uns après les autres une institution au sein de laquelle ils ne trouvaient plus leur place.

\section{NOTES}

1.L'auteur remercie Anne Monjaret de sa coopération dans la rédaction de cet article.

2.Pour une analyse approfondie de l'histoire du musée-laboratoire, voir Martine Segalen, Vie d'un musée 1937-2005, Paris, Stock, 2005

3.Marcel Maget, Ethnographie métropolitaine. Guide d'étude directe des comportements culturels, Paris, Civilisations du Sud, 1953.

4.Grâce à lui sont conservées les affiches dont les étudiants des Beaux-Arts ornèrent les murs de Paris en mai 1968.

5.En réalité neuf, car le tome 6 a été dédoublé, et une « carte et catalogue » des montagnes s'ajoute à l'ensemble. De plus, le CNRS accepta de publier un volume pirate dans lequel les auteurs mettent en doute les conclusions des sociologues publiées dans le tome I. Temps heureux où l'accès au service des publications du CNRS relevait encore d'une organisation artisanale, à dimension humaine.

6.Arch. ATP Rapport à Jean-Paul Lebeuf, 25 janvier 1965.

7.Isaac Chiva est nommé en 1954, Louise Alcan en 1955, Jean-Michel Guilcher en 1958, Mariel Jean-Brunhes Delamarre en 1959 ; Geneviève Vayssière (archives) en 1962, Denise Gluck en 1964, Alain Guey (photographe) en 1965.

8.Ce fut généralement le cas, mais pas toujours. Ainsi Jacques Gutwirth, spécialiste d'anthropologie religieuse bien connu trouvera tout à fait normal de consacrer quelques heures par semaine au département du costume.

9.Ainsi le CNRS accorda-t-il en 1962 un collaborateur technique pour travailler à l'ancien office de la documentation désormais rebaptisé Service de documentation, riche de 3881 manuscrits, 37590 calques, plus de 100000 clichés, et qui jouait un rôle scientifique et culturel de première grandeur dans la maison.

10.Arch. ATP Communication devant la section de muséologie du VII ${ }^{\mathrm{e}}$ congrès international des sciences anthropologiques et ethnologiques tenu à Moscou en août 1964. Le texte décrit les formes de la collecte, en relation avec les démarches de préhistoriens ou d'archéologues : il faut savoir rechercher les « unica » comme les "typica » pour constituer des séries limitées et raisonnées pour fin de constitution de types, sachant que le musée peut consentir des dépôts à des musées de province. En 1961, le musée compte 59280 objets, 70500 imprimés, 11000 phonogrammes, plus de 100000 documents photographiques dont près de 90000 avec leurs clichés, 37500 calques, 1634 monographies d'architecture rurale, 13784 monographies de meubles régionaux, et des cartes postales innombrables.

11.Rapport du 9 octobre1961 intitulé « Politique d'accroissement des collections ». Il s'agit de la première version du rapport du 7 décembre 1961.

12.Arch. ATP His, rapport de 1964. 
13.Aux côtés de tous les membres fondateurs de l'ethnologie française que sont Claude Lévi-Strauss, Georges Condominas, Germaine Dieterlin, Jean Guiart, Jean-Paul Lebeuf, Jean Rouch, Henri Vallois. En 1967, la commission deviendra la section 23.

14.Souvent par le biais des expositions temporaires dont ils eurent la charge, comme ce fut le cas pour « Bergers de France » réalisée par Mariel Jean-Brunhes Delamarre.

15.Contribution de Georges-Henri Rivière au rapport de conjoncture de la section $20 \mathrm{du}$ CNRS (ethnologie).

16.La convention d'association par le Directoire du CNRS est approuvée en décembre 1965.

17.Georges-Henri Rivière avait adressé une lettre à Pierre Monbeig, le 27 décembre 1965 « à vous qui êtes la clef de ce succès, je dis ma très chaleureuse, ma très profonde gratitude ».

18.Christian Bromberger, « Du grand au petit. Variations des échelles et des objets d'analyse dans l'histoire récente de l'ethnologie de la France », in Isaac Chiva et Utz Jeggle (sous la direction de) Ethnologies en miroir. La France et les pays de langue allemande, Paris, Éditions de la Maison des sciences de l'Homme, 1987, p. 67-94.

19.Pour ne reprendre que plus récemment sous l'égide d'un autre conservateur qui choisit de l'aborder sous l'angle des pratiques professionnelles des métiers de la bouche.

\section{INDEX}

Mots-clés : ethnologie, laboratoire, conservateur, ethnologie française, Musée de l'Homme, Centre d'ethnologie française, ethnologie scientifique, ethnographe, musée, arts et traditions populaires, direction des musées de France

\section{AUTEUR}

\section{MARTINE SEGALEN1}

Martine Segalen, actuellement professeur au département de sociologie de l'université de Paris X-Nanterre, est entrée au Musée des arts et traditions populaires (ATP) en 1967 pour seconder Mariel Brunhes-Delamarre dans la publication des volumes Aubrac; elle fut recrutée au Centre d'ethnologie française (CEF) en 1972 pour travailler sur les questions de parenté en milieu rural. Elle dirigea le CEF de 1986 à 1996. 